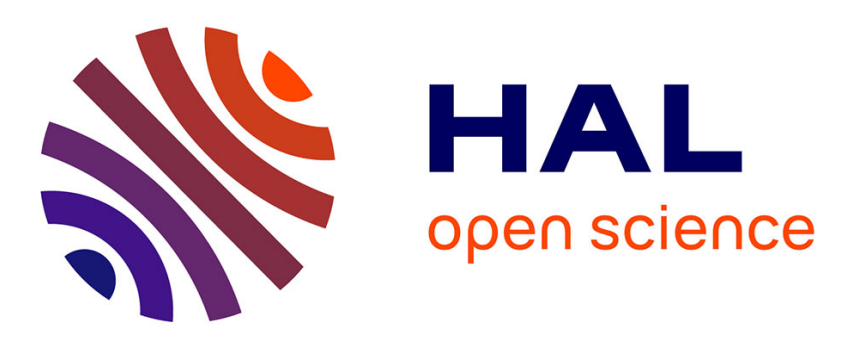

\title{
Benefits of the Adaptive algorithm for retracking altimeter nadir echoes: results from simulations and CFOSAT/SWIM observations
}

Cedric Tourain, Fanny Piras, Annabelle Ollivier, Danièle Hauser, Jean-Christophe Poisson, François Boy, Pierre Thibaut, Laura Hermozo, Céline Tison

\section{To cite this version:}

Cedric Tourain, Fanny Piras, Annabelle Ollivier, Danièle Hauser, Jean-Christophe Poisson, et al.. Benefits of the Adaptive algorithm for retracking altimeter nadir echoes: results from simulations and CFOSAT/SWIM observations. IEEE Transactions on Geoscience and Remote Sensing, 2021, 59 (12), pp.9927-9940. 10.1109/TGRS.2021.3064236 . hal-03026814v2

\section{HAL Id: hal-03026814 \\ https://hal.science/hal-03026814v2}

Submitted on 9 Mar 2021

HAL is a multi-disciplinary open access archive for the deposit and dissemination of scientific research documents, whether they are published or not. The documents may come from teaching and research institutions in France or abroad, or from public or private research centers.
L'archive ouverte pluridisciplinaire HAL, est destinée au dépôt et à la diffusion de documents scientifiques de niveau recherche, publiés ou non, émanant des établissements d'enseignement et de recherche français ou étrangers, des laboratoires publics ou privés. 


\title{
Benefits of the Adaptive algorithm for retracking altimeter nadir echoes: results from simulations and CFOSAT/SWIM observations
}

\author{
C. Tourain, F. Piras, A. Ollivier, D. Hauser, JC. Poisson, F. Boy, P. Thibaut, L. Hermozo, C. Tison
}

\begin{abstract}
The accuracy of sea surface parameters retrieved from altimeter missions is predominantly governed by the choice of the so-called "retracking" algorithm, i.e. the model and inversion method implemented to obtain the surface parameters from the backscattered waveform. For continuity reasons, the choice of space agencies is usually to apply the same retracker from one satellite mission to the other to ensure long time homogeneous series. Here, taking the opportunity of a new configuration of the nadir pointing measurements on-board the recently launched CFOSAT satellite with the SWIM (Surface Waves Investigation and Monitoring) instrument (Hauser et al, 2020), the retracking method was upgraded, by implementing a novel algorithm, called "Adaptive" retracker. It combines the improvements brought by Poisson et al (2018) for the estimation of surface parameters from peaked waveforms over sea-ice, improvements in the way the instrumental characteristics are taken into account in the model (mispointing, point target response) and a more accurate consideration of speckle statistics. In this paper, we first show from simulations carried out in the instrumental configuration of SWIM that the Adaptive algorithm has better accuracy and performances than the classical MLE4 algorithm. Then, the geophysical parameters obtained with real data from SWIM are analyzed with comparisons to reference data sets (model and products from altimeters). We show that this new algorithm has several benefits with respect to the classical MLE4 method: no need of look-up tables to correct biases, significant noise reduction on all geophysical variables especially the significant wave height, and performance of inversion over a large set of echo shapes, resulting from standard oceanic scenes as well as highly specular conditions such as over bloom or sea-ice.
\end{abstract}

Index Terms - retracking algorithm, CFOSAT, SWIM, validation, nadir, altimetry, radar, ocean

\section{INTRODUCTION}

In satellite altimetry, the return echo results from a series of pulses reflecting the Earth's surface. Geophysical variables are obtained from the on-ground processing by inversion of the backscattered echo, using a method called " retracking ». For observations over the sea surface, this method fits an analytical model described by [1] as the convolution of a Point Target Response (PTR), a Flat Sea Surface Response (FSSR) and the Probability Density Function (PDF) of the scattering elements of the elevations. Historically, the first retracking method used for oceanic surfaces was the so-called MLE3 [2] providing three parameters: the epoch, defined as the position of the signal in the analysis window with respect to the tracking reference point (which is then converted in sea surface height $\mathrm{SSH}$ ), the normalized radar cross-section $\sigma^{0}$, and the significant wave height SWH. After the launch of Jason-1 in 2001 and to better account for attitude effects, the MLE4 was introduced [3], providing the same 3 parameters as the MLE3, plus the slope of the trailing edge of the waveform. However, these retracking algorithms show several known limitations. The major one is the need for look-up tables [4] to compensate the error made by modeling the point target response by a Gaussian function. Furthermore, the likelihood function used in the estimator is equivalent to an unweighted least square estimator $[2,4]$. This means that the optimization does not properly account for the speckle noise statistics impacting the waveform and introduces significant noise on the retrieved parameters.

The state of arts of retracking methods also covers improvements dedicated to non-water surfaces. ICE-1 is based on the Offset Centre Of Gravity (OCOG) method [5]; it proves robust for nonstandard waveforms such as those frequently encountered over continental waters [6]. Its outputs are limited to range and $\sigma^{0}$. ICE-2 [7] is based on the fitting of a simplified Brown model [1] around the leading edge for classical ocean geophysical parameter, and ice and land-oriented parameters estimation based on the trailing of the waveform. It provides information over land and ice surfaces. However, over ocean, performances are lower than ocean oriented retrackers (such as MLE3 and MLE4). ALES [8] is designed to be applied both over open and coastal ocean, as it adapts the width of the subwaveform according to the SWH.

A new algorithm, named "Adaptive", was developed by [9] for ENVISAT data processing to improve the continuity of performances of sea level inversion between open ocean and arctic leads. One of its key advantage is the introduction of the mean square surface slope of the dominating reflective surface $(\underline{m s s})$ as a parameter influencing the trailing edge of the echo. This better constrains the retrieval of the normalized backscatter (which is related to mss in specular backscattering conditions) and provides better fits on echoes from highly reflective surfaces such as sea-ice. Finally, the estimation procedure proposed in [9] allows to better account for speckle noise characteristics. This is important for all altimeter missions but even more important for SWIM because of its relatively lower sampling rate of downloaded waveform $(4.5 \mathrm{~Hz}$ instead of $20 \mathrm{~Hz}$ in standard altimeter missions) and hence lower spatial resolution and noisier compressed $1 \mathrm{~Hz}$ data.

In this paper, we propose a modified version of the Adaptive retracking method of [9], by extending its model to take into account the real Point Target Response (PTR) of the altimeter measured in-flight. We also propose another improvement on the approach of [9] by using the mispointing as an input variable, motivated by the specific configuration of the SWIM instrument on board the recently launched CFOSAT mission 
[10,11], which provides a systematic estimation of mispointing angles estimated from the off-nadir observations that can be directly used for the nadir pointing observations Adaptive retracking.

This paper aims at demonstrating the interest and showing the performances of this algorithm. It is illustrated with CFOSAT/SWIM nadir data, but conclusions are more general. In the following, we will present a performance analysis of this novel Adaptive retracking algorithm based on both simulated and real data sets.-As the SSH is not a variable to be provided to users in SWIM products, this paper focuses on SWH and $\sigma^{0}$. It is organized as follows: in section II, the main characteristics of SWIM and its raw data are summarized. In section III we recall the theoretical background of the current retracking algorithms (used in ground-segments of space agencies), and present the modifications brought by the Adaptive retracking. Then in section IV, simulation results obtained for an observation configuration similar to SWIM are discussed to illustrate the improvements brought by the Adaptive Algorithm, in comparison to the MLE4 algorithm. In section V, the performances of the Adaptive Algorithm are assessed on SWIM real data by comparing results from the Adaptive and the MLE4 methods. In section VI, SWIM geophysical products obtained with the Adaptive algorithm are compared to model outputs and altimeter products at cross-over points to assess their performance with respect to independent data sets. We finally conclude and give perspectives in the last section.

\section{SWIM NADIR MODE CONFIGURATION}

The CFOSAT program [10] is carried out through a cooperation between the French and Chinese Space Agencies (CNES and CNSA respectively). It aims at characterizing the ocean surfaces to better model and predict the ocean states and improve the knowledge in ocean/atmosphere exchanges. Data over continental surface are also available for studies on continent.

The CFOSAT satellite was launched on 2018 October $29^{\text {th }}$ with on-board two scientific Ku-Band radars: SWIM, a nadir and near-nadir wave scatterometer [11] and SCAT, a wind scatterometer [12]. CFOSAT has a sun synchronous orbit repetitive with a 13-day cycle, its altitude and inclination are respectively $520 \mathrm{~km}$ and $97.4^{\circ}$ Although CFOSAT is not a standard altimeter mission, SWIM instrument includes a nadir pointing designed to measure SWH and $\sigma^{0}$ as classically carried out from other altimeter missions. In contrast, neither the epoch nor SSH, was specified as a variable to be provided to users. SWIM has also five beams pointing near nadir to estimate directional spectra of ocean waves $[11,13]$. The six beams illuminate the surface sequentially at $0^{\circ}, 2^{\circ}, 4^{\circ}, 6^{\circ}, 8^{\circ}$ and $10^{\circ}$ incidence with respect to nadir, with a scanning geometry (see $[11,13])$. Due to this specificity the nadir waveform sampling is slightly different from standard altimeter missions: although the number of raw samples considered in the on-board integrated echo is larger than that of standard altimeter missions (264 instead of 90 for Jason missions), the mean waveform after on-board processing is provided at a $4.5 \mathrm{~Hz}$ rate, instead of the usual $20 \mathrm{~Hz}$ in standard altimetry. This is because time is reserved for the acquisition of the signal on the nonnadir beams. This means that the native data cannot be analyzed at high $(\sim 20 \mathrm{~Hz})$ frequency, and that the noise on the postprocessed mean values provided at $1 \mathrm{~Hz}$ is larger than for other altimeter missions (for a same instrument characteristics). Therefore, specific effort must be done to avoid additional noise on the retrieved parameters due to the inversion.

Another feature of SWIM on-board CFOSAT is that the mispointing angle is provided, as an ancillary information, by the off-nadir beam at the same frequency as the waveform ( 4.5 $\mathrm{Hz}$ ). The method to estimate the mispointing is explained in [13]. This information is particularly important for SWIM as it was shown [13] that the mispointing varies slightly with the look angle during the rotation of the feed horn plateau.

\section{THEORETICAL BACKGROUND ON NADIR RETRACKING AND DESCRIPTION OF THE ADAPTIVE METHOD}

The general principle of usual retracking algorithms [1] is to fit a waveform model to the real signal received by the instrument. The inversion (here-after called estimation procedure) is carried out by using minimization of a likelihood function, which characterizes the distance between model and observations. In this section, we recall the historical background for both the MLE4 and the Adaptive method and describe the choices for the inversion procedure.

\section{A. Theoretical background and MLE4 echo model}

Here we first describe the theoretical background for the model used in the commonly used retracker for oceanic surfaces (MLE4), then the specificities of the Adaptive model are detailed in section III B.

The signal $S(t)$ received by the instrument is given by the following double convolution $[1,15]$ :

$$
\mathrm{S}(\mathrm{t})=\operatorname{FSSR}(\mathrm{t}) * \operatorname{PDF}(\mathrm{t}) * \operatorname{PTR}(\mathrm{t})(1)
$$

where FSSR is the flat sea surface response, PDF is the surface elevation probability density function of scattering elements, PTR is the radar system point target response and $*$ is a convolution product.

\section{1) Probability Density Function: PDF(t) function}

The surface elevation probability density function of scattering elements, is given by Eq I.28 in [16], considering a fourth order development:

$$
P D F(\eta)=\frac{1}{\sqrt{2 \pi \sigma_{s}^{2}}} \exp \left(-\frac{\eta^{2}}{2}\right)\left[1+\frac{\lambda s}{6}\left(\eta^{2}-3 \eta\right)+\frac{K s}{24}\left(\eta^{4}-6 \eta+3\right)\right]
$$

$\eta$ being the height normalized by the standard deviation of wave heights $\sigma_{s}$ [16]. It is also characterized by the skewness $\lambda_{s}$ (third order moment) and the kurtosis $K_{s}$ (fourth order moment). Brown [1] approximates this expression by a Gaussian model with $\lambda_{s}=0$ and $K_{s}=0$. Hayne [15] uses a non-null skewness coefficient $\lambda_{s}$. 


\section{2) Point Target Response: PTR(t) function}

The radar point target response is generally expressed as a square sinc function that results from the deconvolution of the emitted chirp (linear modulation of the frequency w.r.t. the time) [3]:

$$
\operatorname{PTR}(t)=\left|\frac{\sin \left(\pi \frac{t}{T s}\right)}{\pi \frac{t}{T s}}\right|^{2}
$$

Where $T s=1 / B$ is the sampling period and $B$ the radar system reception bandwidth.

Brown [1] approximates this expression by a Gaussian model which according to $[3,16]$ leads to:

$$
\operatorname{PTR}(\mathrm{t})=\frac{1}{\sqrt{2 \pi} \sigma_{\mathrm{p}}} \exp \left(\frac{-\mathrm{t}^{2}}{2 \sigma_{\mathrm{p}}^{2}}\right)
$$

With $\sigma_{p}=0.513 T s$ related to the PTR width at $-3 \mathrm{~dB}$ [17].

\section{3) Flat sea surface response model: FSSR(t) function}

The final formulation of the model commonly used in conventional altimetry is derived from the Brown model [1]. In this formulation, the flat sea surface impulse response in (1) is given by:

$$
F S S R(t)=A P \exp \left[-\frac{c t}{h}\left(\frac{4}{\gamma} \cos 2 \xi+\alpha\right)\right] I_{0}\left(\beta t^{1 / 2}\right)
$$

Where:

$$
\begin{array}{ll}
\text { - } & \mathrm{A}=\exp \left(\frac{-4 \sin ^{2} \xi}{\gamma}\right) \\
\text { - } & \gamma=\frac{2}{\ln (2)} \cdot \sin ^{2}\left(\frac{\theta_{0}}{2}\right) \text { with } \theta_{0}: 3 \mathrm{~dB} \text { antenna beamwidth } \\
\text { - } & \text { P: amplitude of the signal } \\
\text { - } & \text { h: satellite altitude } \\
\text { - } & \xi \text { is the absolute off-nadir pointing angle } \\
\text { - } & \alpha \text { is a function of the radar observed surface } \\
& \text { characteristics, mainly the mean square slope. } \\
\text { - } & I_{0}: \text { Bessel function } \\
\text { - } & \beta=\frac{4}{\gamma}\left(\frac{c}{h}\right)^{1 / 2} \sin 2 \xi
\end{array}
$$

Haynes [15] ignored the $\alpha$ parameter, assuming that sea surface roughness cannot be null. The expression is then simplified from (5) to (6).

$$
F S S R(t)=A P \exp \left[-\frac{c t}{h}\left(\frac{4}{\gamma} \cos 2 \xi\right)\right] I_{0}\left(\beta t^{1 / 2}\right)
$$

The final formulation of the return power, considering the skewness coefficient and a Gaussian approximation of the PTR is an analytical model described in [15] and [16] and is the one used in the ground-segments of space agencies for the MLE4 algorithm.

\section{B. The Adaptive echo model}

The formulation proposed in [15] shows limitations in the case of sea ice and leads. Based on the FSSR model developed by Amarouche ([18, 19]), a modified formulation of the FSSR was proposed in [9]:

$$
F S S R(t)=A P \exp \left[-\frac{c t}{h}\left(\frac{4}{\Gamma}\right)\right] I_{0}\left(\beta t^{1 / 2}\right)
$$

Where $\quad \Gamma=\frac{4 \gamma \mathrm{mss}}{4 \mathrm{mss}+\gamma}$ and mss: mean square slope (7a)

The main evolution in this model with respect to the most classical expression of (6) is that surface characteristics effects are taken into account through the mss parameter in the exponential parameter (and not only through the amplitude of the signal), allowing a far greater flexibility to adapt to specular echoes (see section IV). Indeed, contrary to the Haynes model (6) that constrains its trailing edge with the off-nadir angle only, this approach allows the trailing edge of the echo to be also constrained by the surface roughness.

To do so, the Amarouche model uses the following $\sigma^{0}$ modeling:

$$
\sigma^{0}(\theta)=\sigma^{0}(0) \exp \left(-\frac{\sin ^{2}(\theta)}{m s s}\right)
$$

Where $\sigma^{0}$ is the normalized cross-section, proportional to the amplitude $\mathrm{P}$ of the signal, $\theta$ is the incidence angle from nadir and $m s s$ the mean-square surface slope of the dominating reflective surface in the altimeter footprint [9]. However, this formulation, as used in [9] does not take into account the offnadir angle, contrary to the original Brown model [1].

Therefore, we propose to use the full expression of the Adaptive model as described in [18], allowing to specify the mispointing as an input. (7) stays as it is but with the $\Gamma$ parameter replaced by:

$$
\Gamma=\frac{4 \gamma \mathrm{mss}}{4 \mathrm{mss} \cos 2 \xi+\gamma}
$$

(9) is equivalent to (7a) when the mispointing angle is neglected.

As explained in [9], it is assumed that even the most reflective surfaces such as sea-ice leads have a non-null surface roughness, meaning that the parameter $m s s$ in (9) can never be equal to zero.

Finally, the formulation of the analytical model used in the Adaptive is reduced to the following Sea Surface Response (SSR):

$$
\begin{gathered}
\operatorname{SSR}(\mathrm{t})=\frac{\mathrm{A} \sigma_{0}}{2}\left[1+\operatorname{erf}\left(\frac{t-\tau-\frac{4 c}{\Gamma h} \sigma_{\mathrm{s}}{ }^{2}}{\sqrt{2} \sigma_{\mathrm{s}}}\right)\right] \\
\mathrm{x} \exp \left[-\frac{4 c}{\Gamma h}\left(t-\tau-\frac{2 c}{\Gamma h}{\sigma_{\mathrm{s}}}^{2}\right)\right]+N_{t}
\end{gathered}
$$

Where: $\tau$ is the epoch, and $\mathrm{Nt}$ is the additive thermal noise.

Compared to Eq. (5) of [9], the composite variable $\sigma_{c}$ of [9] $\left(\sigma_{c}=\left(\sigma_{s}{ }^{2}+{\sigma_{p}}^{2}\right)^{1 / 2}\right)$ is replaced by $\sigma_{s}$, by imposing a null value of the PTR-related variance $\sigma_{p}{ }^{2}$. The analytical model 
expression is reduced to the only convolution of the FSSR with the PDF, called Sea Surface Response. The model expression (1) can then be expressed as the convolution of the PTR with SSR: $S(t)=\operatorname{SSR}(t) * \operatorname{PTR}(t)$. In the context of the Adaptive retracking, this convolution is performed numerically.

The final formulation of the model used in the Adaptive retracking, here after called the "Adaptive model" is therefore a semi-analytical model, based on the analytical expression (10) and a numerical PTR.

\section{C-Likelihood function and minimization procedure}

The most commonly used minimization procedures are based on a likelihood estimator (like MLE), in order to take into account noise associated to the data in the inversion process. Indeed, radar data is corrupted by a multiplicative speckle noise. To reduce the impact of this noise affecting each individual echo, a sequence of consecutive pulses is averaged on-board. Assuming pulse-to-pulse statistical independence, the resulting speckle noise follows a Gamma distribution depending on the number $\mathrm{N}$ of averaged individual pulses. A likelihood function can be derived from these statistics [20], and is expressed as:

$$
C=c s t+N \sum_{k=0}^{K-1} \frac{y_{k}}{s_{k}}-(N-1) \sum_{k=0}^{K-1} \ln \left(y_{k}\right)+N \sum_{k=0}^{K-1} \ln \left(S_{k}\right)(11)
$$

Where $y_{k}$ is the measured waveform, $S_{k}$ the model, $k$ the waveform sample number within $\mathrm{K}$ samples, and cst a constant.

In common conventional altimetry processing such as the MLE4, a simplification of (11) is often applied following expression (12) which is a more classical least square estimator.

$$
C=\sum_{k=0}^{K-1}\left(y_{k}-S_{k}\right)^{2}(12)
$$

This is not optimal as it does not account for noise properties of the measured variable and this can have an impact on the final noise on the retrieved parameters.

\section{D- The Adaptive algorithm for CFOSAT/SWIM}

The first specificity of the algorithm selected for SWIM is to choose the Adaptive echo model from (10) with the parameter $\Gamma$ given by (9), considering a Gaussian model for the surface elevation probability density function (null skewness $\lambda_{\mathrm{s}}$ and kurtosis $K_{s}$ in (2)). When applied on SWIM real data, the mispointing angle $\xi$ is fixed from off-nadir angle estimations provided independently from the off-nadir beam observations of SWIM (see [13]). Parameters estimated are thus: the epoch $\tau$, the standard deviation of wave heights $\sigma_{s}$, the amplitude of the signal $P$ and the mean square slope $m s s$.

The second specificity of the algorithm proposed here is that we choose to use the real in-flight PTR of the instrumental, by convolving its discretized values numerically to the analytical model given by (10).

We will see further below that this choice is significant as it enables to estimate the geophysical parameters without any bias correction to compensate from the Gaussian approximation of the PTR. Furthermore, it can take into account the natural ageing of the instrument and its potential effects (sides lobe dissymmetry, evolution of the position of the maximum of the PTR, .. ). Indeed, for SWIM as for most altimeters, the PTR is measured regularly through an internal calibration sequence mode [13]. Therefore, the PTR used as input of the Adaptive is updated along with the calibration sequences, to follow the instrument's ageing as close as possible.

The third specificity is to minimize the model by using the likelihood estimator (MLE) from (11), i.e. taking into account the noise properties of the radar echo. This is different from most of the MLE4 algorithms implemented for the current altimeter missions, which use the simpler least square estimator (LSE) given by (12).

A numerical scheme is required to invert the statistical estimator function. Two main algorithms are used in conventional altimetry. The first one is the Newton-Raphson algorithm. It minimizes the cost function by a gradient descent approach. The second algorithm is a geometrical method called the Nelder-Mead algorithm [21]. It is a direct search method (based on function comparison) and is often applied to nonlinear optimization problems for which derivatives may not be known.

The Newton-Raphson algorithm has the advantage of converging in a reduced number of iterations thanks to the gradient descent method. To do so, the cost function and its derivatives must be estimated. The Nelder-Mead algorithm needs more iterations, however only the cost function must be estimated, which simplifies the implementation and reduces the number of operations at each iteration.

Therefore, for SWIM nadir processing, the Nelder-Mead method has been selected as the optimization algorithm.

In the SWIM products, the outputs of this inversion are the SWH estimated from the $\sigma_{\mathrm{s}}$ parameter of (10) and the normalized radar backscatter $\sigma^{0}$, constrained by both the received power $P$ and the $\Gamma$ parameter of (6).

The improvements brought by each of these evolutions with respect to the classical MLE4 algorithm are detailed in the following sections.

\section{THEORETICAL BENEFITS OF THE ADAPTIVE ALGORITHM BASED ON SIMULATIONS}

In this section, a simulated dataset is used to assess the proposed method based on (10-11) and to compare its performance to the more classical retracking algorithms. We consider four configurations, as detailed in

Table 1 below.

\begin{tabular}{|c|c|c|c|}
\hline Name & Model & PTR & $\begin{array}{c}\text { Likelihood } \\
\text { criterion }\end{array}$ \\
\hline MLE4 & Brown & $\begin{array}{c}\text { Gaussian } \\
\text { Model }\end{array}$ & LSE \\
\hline R1 & Brown & Real & LSE \\
\hline R2 & Brown & Real & MLE \\
\hline Adaptive & Adaptive & Real & MLE \\
\hline
\end{tabular}

Table 1: List and specificities of the different retracking solutions considered in the trade-off. 


\section{A. Simulated data set and method}

The dataset was generated by modeling waveforms based on the analytical Hayne model according to (6) convolved with a real point target response (PTR). This latter was taken from a measured PTR of SWIM dated from August 27 ${ }^{\text {th }}$, 2019. The simulations parameters are based on the CFOSAT geometry and SWIM instrumental characteristics. They are given in Table 2. Note that no mispointing is considered in these simulations. Here we generate directly $4.5-\mathrm{Hz}$ averaged waveforms, so the speckle noise is applied to model waveforms through a Gamma law $(1 / \mathrm{N}, \mathrm{N})$ with $\mathrm{N}=264$, corresponding to the real number of SWIM samples accumulated at this $4.5 \mathrm{~Hz}$ frequency. Simulations are generated for different SWH values, from 1 meter to 8 meters, with a step of 1 meter. Roughness is considered as homogeneous in the footprint. For each step, a random draw of 10000 gamma laws is generated and then multiplied to the modelled waveform. The thermal noise being an additive noise, it is thus applied posterior to the speckle noise multiplication step.
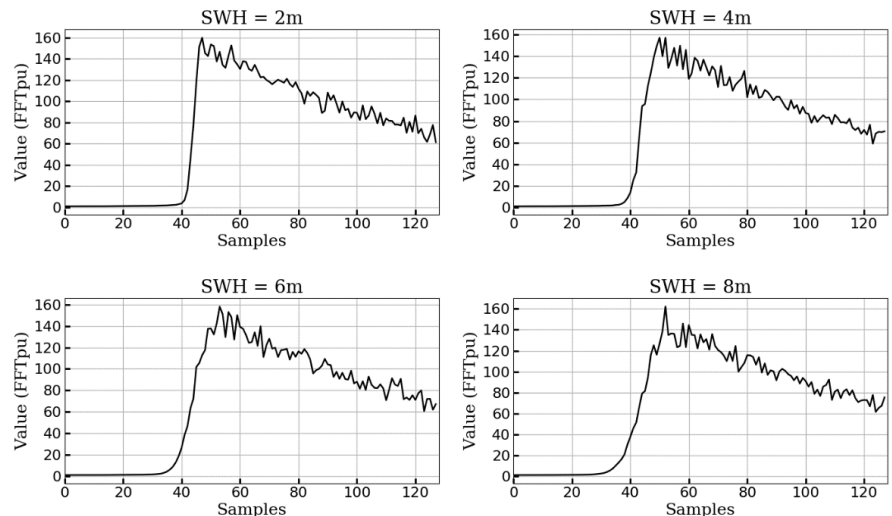

Figure 1: Example of waveforms simulated for four SWH steps $(2 \mathrm{~m}$, $4 m, 6 m$ and $8 m$ ), after application of speckle noise and thermal noise. The Y axis represents the amplitude in FFT power units.

\begin{tabular}{|l|c|l|c|}
\hline Altitude (Km) & 550 & Amplitude (p.u) & 160 \\
\hline Bandwidth (Hz) & 320 & Thermal Noise (p.u) & 1.0 \\
\hline Sampling Freq. (Hz) & 400 & Skewness & -0.1 \\
\hline Ant. Beam $\left(^{\circ}\right)$ & 1.51 & ${\text { Off-nadir angle }\left(^{\circ}\right)}^{0}$ & 0 \\
\hline Nb of samples & 256 & First useful gate $^{6}$ & 64 \\
\hline Ref. Abscissa & 108 & Last useful gate & 192 \\
\hline
\end{tabular}

Table 2: Simulation parameters

The resulting datasets are then processed by the four retracking algorithms presented in

Table 1.

The first one corresponds to the MLE4, which is the current operational conventional altimetry retracking algorithm. Then, components are modified one after the other to observe their impact on the estimation performances.

To assess the performances of all three configurations, the following diagnosis are performed:
- Waveform residuals (the average difference between the model and the signal) for each SWH step, to assess the quality of model fitting.

- The average bias per SWH step, i.e. the mean difference between the simulation input parameter and the result, over the 10000 draws.

- The estimation noise per SWH step, i.e. the mean standard deviation over the 10000 draws.

\section{B. Impact of the Point Target response: R1 versus MLE4}

As already described, the conventional operational retracking algorithm considers a modeled point target response, which implies the use of look-up tables [14] to compensate SWH and $\sigma^{0}$ biases. As shown in Figure 2, considering the real point target response through a convolution (method R1, orange curves) instead of a Gaussian model (method MLE4, black curves) improves remarkably the fit of the modeled waveform with the signal.

The differences between fitted echoes and the reference waveforms are significantly smaller for the R1 case (orange curve) than for the MLE4 case (black curve)

This especially visible for the leading edge (samples from 30 to 50). Note also that according to Figure 2, the improvement increases when SWH decreases. Retrieved mean values given in Figure 3a, show that with R1 (orange curve), the bias on the retrieved SWH is very low (less than $1 \mathrm{~cm}$ ), in opposite to MLE4 (blue curve). For MLE4, look up tables are computed (cf.§ V.A) and used to compensate the bias observed. This interestingly shows that when using the real Point Target Response in the convolution product, no more look up tables are needed for correcting this parameter after inversion. As for the mean value of $\sigma^{0}$ (Fig.3b) we observe no significant difference between the MLE4 and R1 solution: a bias of $0.25 \mathrm{~dB}$ is found for both configurations. This was expected because the Gaussian approximation of the PTR have a low impact on this parameter.

\section{Impact of the likelihood function: $R 1$ vs $R 2$}

As illustrated in Figure 3, the implementation of the likelihood function (10) (R2 : orange curve ) induces a $60 \%$ noise reduction on SWH and $11 \%$ on $\sigma^{0}$, with respect to a least square estimator (R1: red curve). The solution with R2 also significantly reduces the bias on $\sigma^{0}$, providing a null bias (compared to $0.25 \mathrm{~dB}$ for $\mathrm{R} 1$ ). Indeed, by using the MLE likelihood criterion, the speckle law statistics is taken into account, allowing not only to reduce the estimation noise, but also to better fit the trailing edge of the waveform, reducing the remaining small $\sigma^{0}$ biases to almost zero. The same effect is expected on the SWH parameter, even though it is not significant enough to be visible on these simulations. Hence, these results show that to be fully free from look-up tables, the introduction of the real PTR is the most appropriate to get rid of SWH bias, while the MLE likelihood criterion is more appropriate to get unbiased $\sigma^{0}$. 

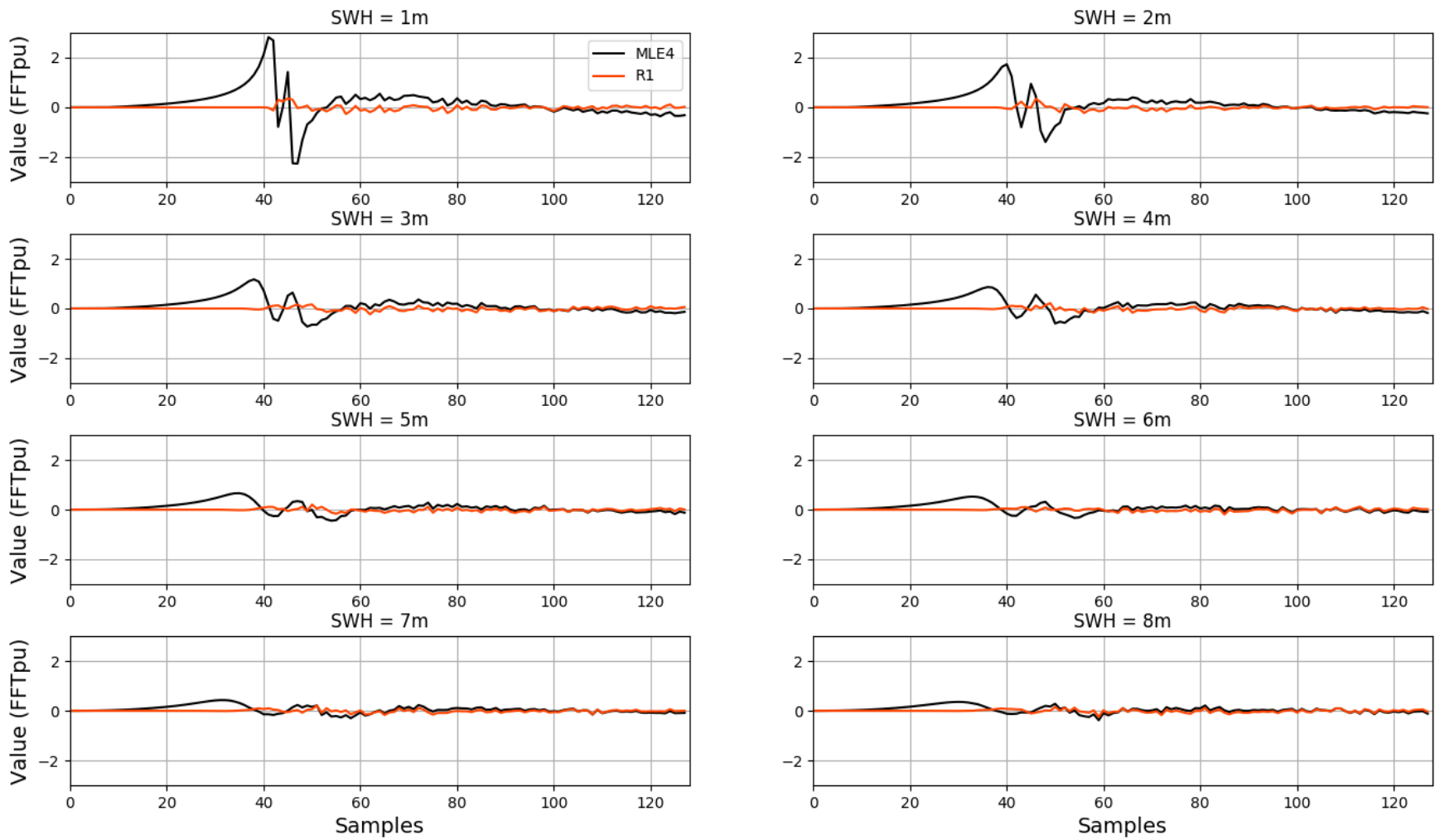

Figure 2 : Differences between fitted echoes and the reference waveforms over all samples for a retracking processing with MLE4 algorithm with modeled PTR convolution (black curve) and R1 retracking with real PTR convolution (red curve). The Y axis represents the amplitude in FFT power units.

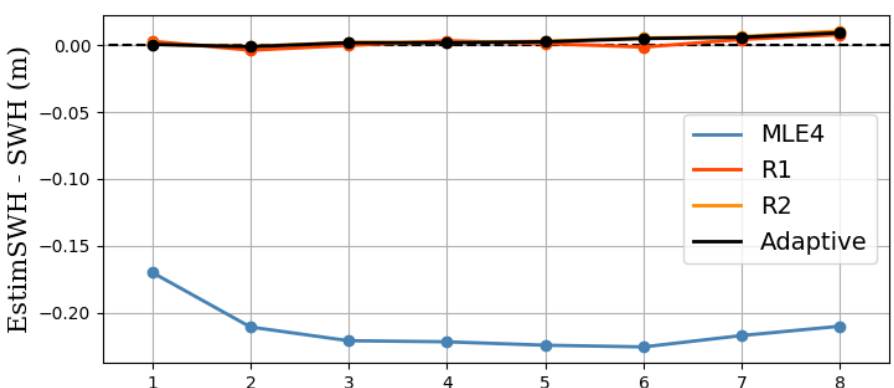

(a)

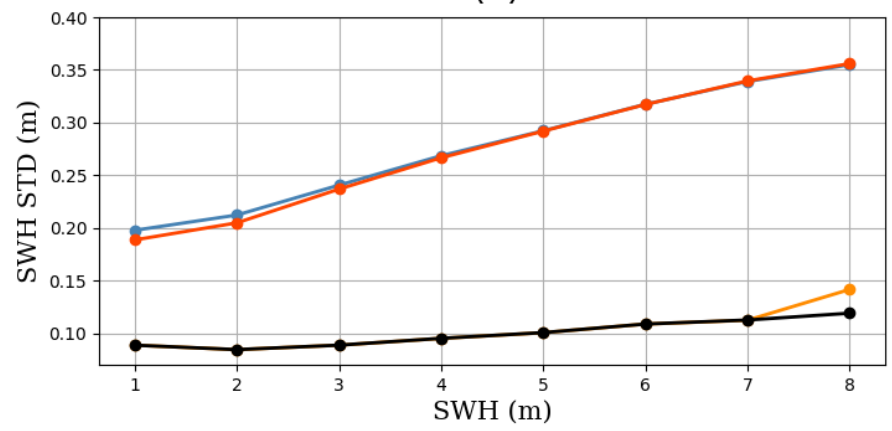

(c)

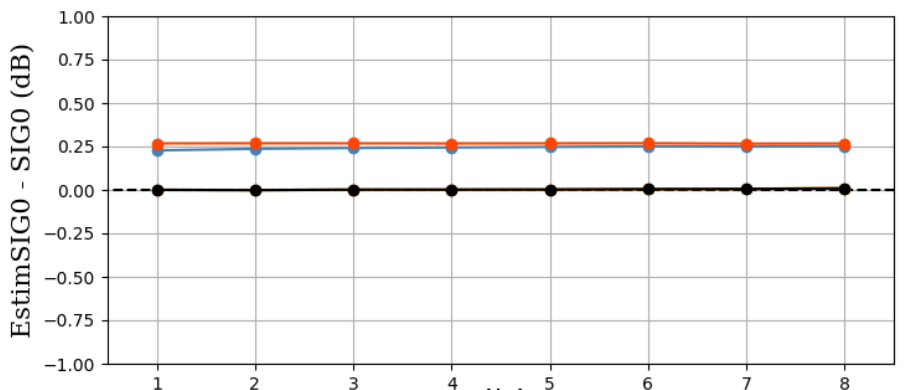

(b)

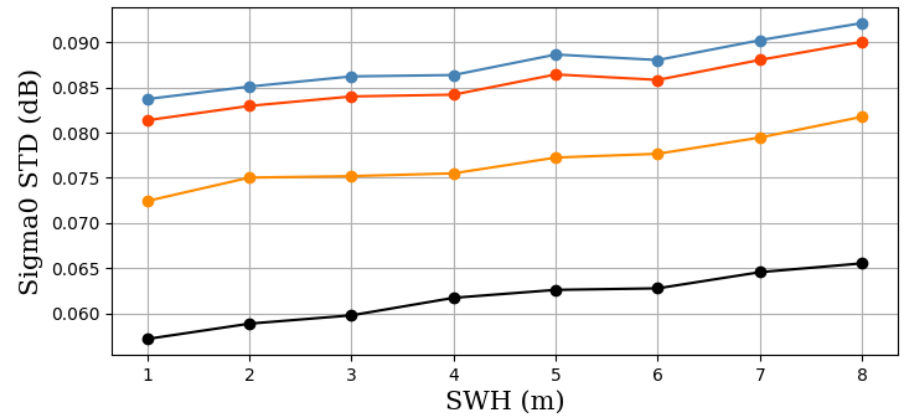

(d)

Figure 3 a) SWH biases b) $\sigma^{0}$ biases c) SWH standard-deviation d) $\sigma^{0}$ standard deviation. Statistics retrieved from MLE4 (blue), R1 (red), R2 (orange) and Adaptive (black) algorithms. Note that in (c), the orange curve for $R 2$ is hidden behind the black one 


\section{Adaptive model vs Brown model: Adaptive vs $R 2$}

Figure 3 shows that the Adaptive (black curves) and R2 solution (yellow curves) show equivalent performances in terms of bias for both SWH and $\sigma^{0}$. This is expected because the main difference between the Adaptive and R2 is the introduction of the $m s s$ in the $\sigma^{0}$ formulation. In the case of classical oceanic echoes such as the simulated dataset used in this section, the introduction of the $m s s$ has negligible impact on the biases.

The main difference is for the $\sigma^{0} \mathrm{rms}$ error where a noise reduction of $20 \%$ is observed compared to R2. As explained just before, this is due to the fact that in the Adaptive model, the $\sigma^{0}$ formulation considers the mss parameter (8); therefore, it is better constrained by the echo shape. In fact, the interest of the Adaptive model with respect to Brown model is the most significant on peaky echoes processing. This is illustrated in Figure 4, which shows a simulated peaky echo and the corresponding models for both MLE4 and Adaptive retracking. The MLE4, aimed at processing ocean echoes, tries unsuccessfully to fit the Brown model, whereas the Adaptive model perfectly fits the waveform, proving its ability to retrack echoes that are not typical ocean diffuse echoes.
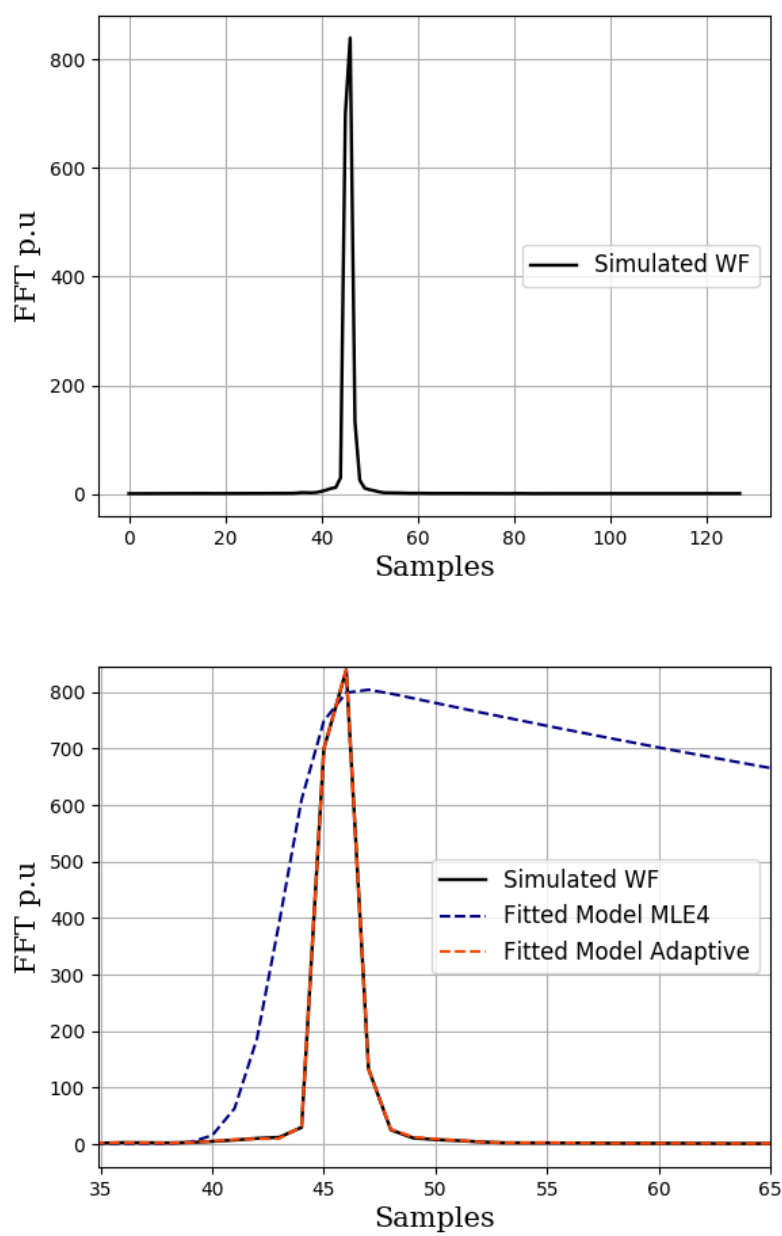

Figure $4:$ A simulated peaky echo (upper plot) and the corresponding models for Adaptive (red) and MLE4 (blue), zoomed on the useful part of the signal (lower plot). The Y axis represents the amplitude in FFT power units.

\section{ADAPTIVE VERSUS MLE4: RESULTS OBTAINED ON REAL CFOSAT DATA}

The different results presented above have led us to the selection of the so called Adaptive retracking algorithm for the SWIM nadir data processing in the NRT CFOSAT ground segment. Note that Adaptive retracking refers below to the configuration described in section III-D combined with the Nelder-Mead algorithm for the minimization process.

In this section, we compare the results obtained from the same SWIM data set using the Adaptive algorithm on one side and the MLE4 algorithm on the other side.

\section{A. Description of the data used}

We use here two different datasets, both obtained on 13 days of CFOSAT data (1 orbital cycle): the first dataset contains Adaptive retracking outputs, obtained directly from the groundsegment products. The second dataset contains MLE4 retracking outputs, obtained by applying off-line the MLE4 processing on the $5 \mathrm{~Hz}$ nadir SWIM echoes, for the purpose of this demonstration. The cycles chosen for this analysis are the cycle 5 and cycle 16 , covering respectively the periods from 2018 December $23^{\text {th }}$ to 2019 January $05^{\text {th }}$ and 2019 May $15^{\text {th }}$ to 2019 May $28^{\text {th }}$.

To consider comparable datasets, SWH look-up tables have been computed to compensate for the error made using a Gaussian approximation of the PTR in the MLE4 retracker. These look-up tables have been computed using the simulations described in section III.A, for SWH values from $0.25 \mathrm{~m}$ to $12 \mathrm{~m}$ with a step of $0.25 \mathrm{~m}$. This correction on significant wave height varies between $15 \mathrm{~cm}$ and $20 \mathrm{~cm}$ (Figure 5) and has been applied to the SWH MLE4 dataset for the analysis presented in this section. Note that no look-up tables have been computed here for the $\sigma^{0}$ parameter, as they are usually considered negligible and not used in the operational MLE4 products.

Results also include comparisons to models, interpolated at SWIM resolution.
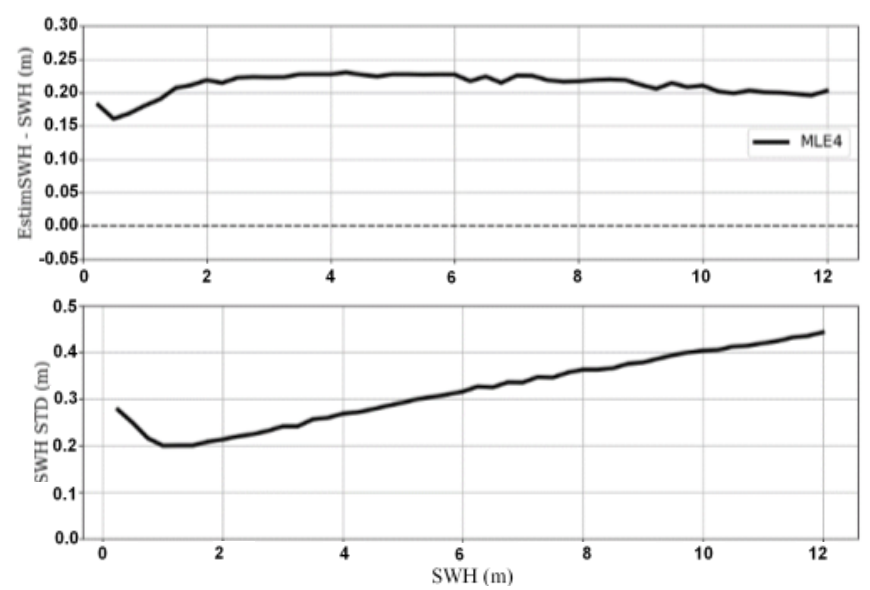

Figure 5: MLE4 Look-Up tables for Significant Wave Height 


\section{B. Fit of the waveform}

To assess the quality of a retracker's waveform fit, the MSE (Mean Squared Error), also called MQE (Mean Quadratic Error) is computed. The comparison of MQE obtained between MLE4 and Adaptive, illustrated in Figure 6, shows the superior Adaptive performances: in average, the Adaptive MQE is $30 \%$ lower than the MLE4 MQE with a similar standard deviation (Fig 6a). The map of the differences (Fig 6b) displays only positive values, meaning that the Adaptive fit is an improvement compared to the MLE4, over all regions of the planet. This improvement is heterogeneous and different patterns appear, correlated with the roughness of the surface, which is a consequence of two main differences in the algorithm. Firstly, by considering the real PTR of the instrument, the fit on the leading edge is largely improved with the Adaptive model compared to MLE4 (Figure 2) in particular at low to moderate sea-state conditions. Secondly, as mentioned before, one interest of the Adaptive method is to better fit nonstandard peaky echoes as observed on highly specular surfaces such as bloom events or marginal ice over the ocean, thanks to the inclusion of a parameter related to the surface characteristics (namely the square slope $\mathrm{mss}$ ) in the exponential term in (10).
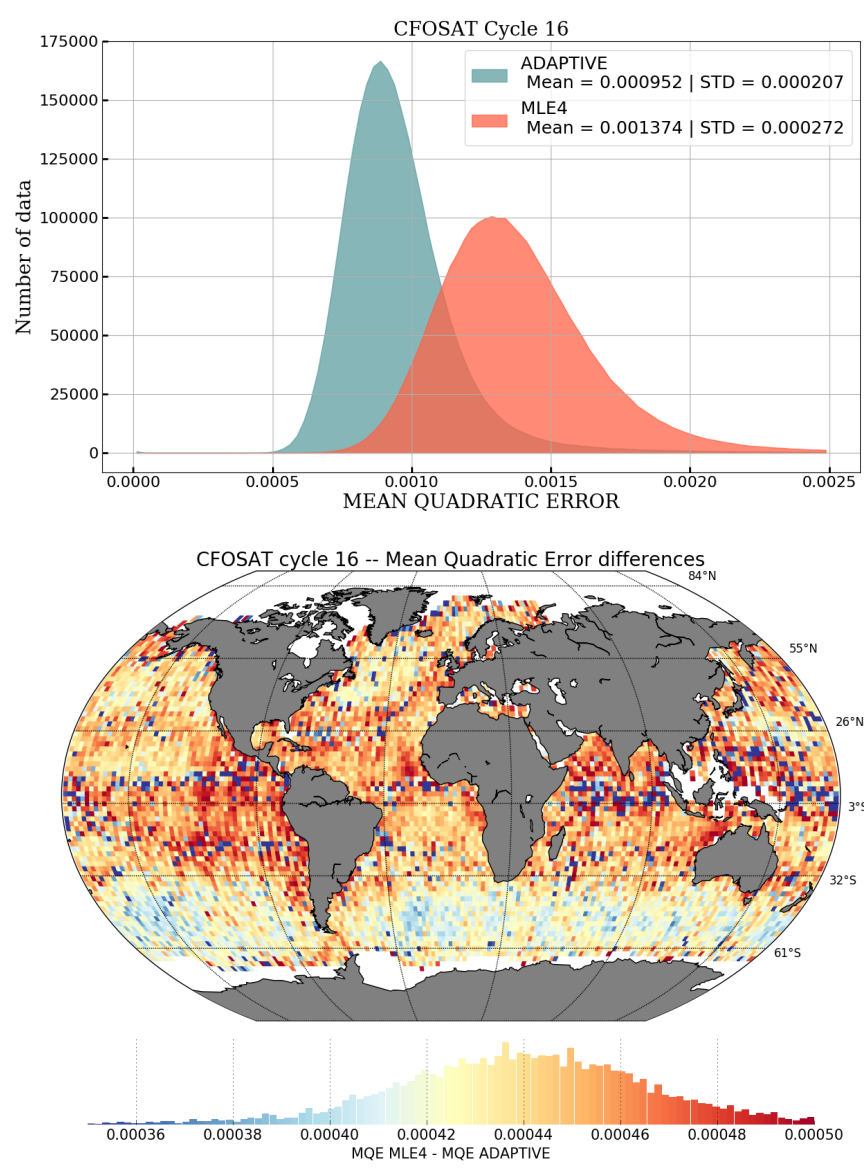

Figure $6:$ Histograms of MQE Adaptive (Blue) and MLE4 (Red) (Upper plot) and gridded map of the difference of $M Q E$ between MLE4 and Adaptive (lower plot) for CFOSAT cycle16

\section{Significant Wave Height}

Figure 7 shows the differences between the SWH parameter (MLE4 or Adaptive) retrieved from altimetry and from the ECMWF model, interpolated at the altimeter resolution, over data from cycle \#16 (13 days). Both solutions show a low mean bias ( $\sim 1 \mathrm{~cm}$ for MLE4 and $\sim 3 \mathrm{~cm}$ for Adaptive), confirming that no look-up tables are needed for the Adaptive. In addition, the Adaptive measurements show a $40 \%$ lower standard deviation compared to the MLE4 solution, which is consistent with the results presented in section IV.

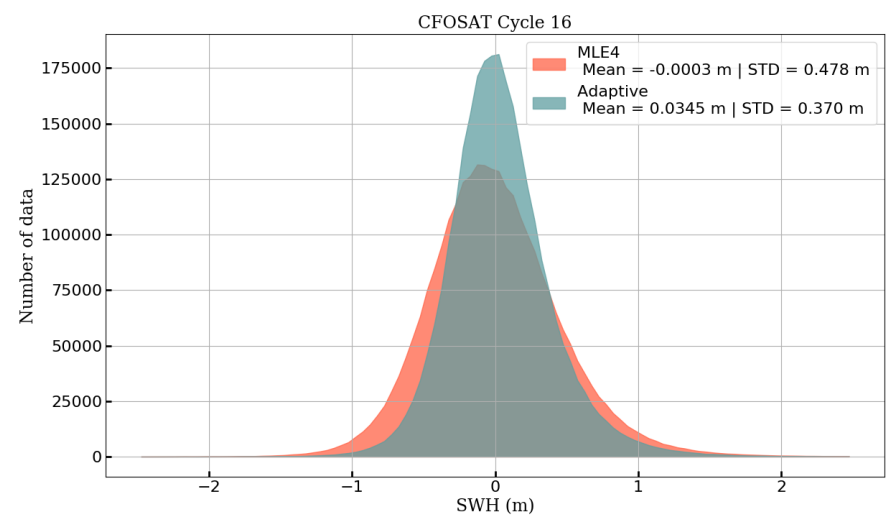

Figure 7: Histograms of SWH differences between MLE4 (LUTS applied) and ECMWF (orange), and Adaptive (No LUTs) and ECMWF (green) for CFOSAT cycle 16

A spectral analysis on the SWH parameter, performed for MLE4 and Adaptive, is illustrated in Figure 8. The spectra are obtained from continuous series of SWH values along track (segments of $700 \mathrm{~km}$ long). Noise levels can be computed using the following formula:

$$
\text { Noise Level }(\mathrm{cm})=\sqrt{\frac{P_{S}}{2 * \Delta \tau}}
$$

Where $\Delta \tau$ is the resolution sampling $(1.4 \mathrm{~km}$ for $5 \mathrm{~Hz}$ CFOSAT data), and $P_{S}$ is the power spectrum computed after the averaging of several individual power spectra obtained on independent data segments [22].

For MLE4, the $5 \mathrm{~Hz}$ noise level is equal to $36.8 \mathrm{~cm}$, whereas the Adaptive $5 \mathrm{~Hz}$ noise level equals to $21.7 \mathrm{~cm}$. Hence, the Adaptive reduces the SWH noise level by $41 \%$ compared to the MLE4 solution. The results obtained on simulations (section IV) predicted a $60 \%$ noise reduction, however these simulations did not take into account the $4.5 \mathrm{~Hz}$ sampling rate of the SWIM products, which is not enough to reach the "instrumental plateau" or white noise, that can be observed on $20 \mathrm{~Hz}$ spectral analysis [23]. A similar comparison, done with Jason-3 data (not shown here), shows a $60 \%$ SWH noise reduction. Nevertheless, the current Jason-3 instrumental noise on the SWH parameter, retrieved with MLE4, is around $50 \mathrm{~cm}$ [23], meaning that SWIM SWH is $60 \%$ less noisy than the current Jason-3 SWH product. 


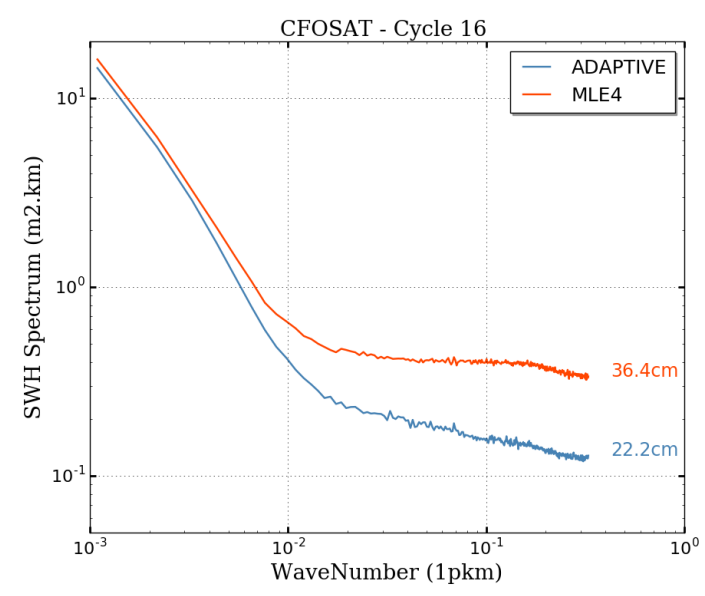

Figure 8: MLE4 and Adaptive SWH spectral analysis (MLE4 in orange, Adaptive in blue). For the MLE4 spectrum, look-up tables are accounted for. Noise levels are computed for both solutions.

\section{Backscattering coefficient $\sigma^{0}$}

Similarly to the along-track spectra shown for SWH in Figure 8 , Figure 9 shows the spectra estimated for the $\sigma^{0}$ parameter. The MLE4 (orange curve) shows a "hump" at specific scales. This is a well-known artefact [23] due to a strong correlation between the slope of the trailing edge and the $\sigma^{0}$ parameter, in conditions when the MLE4 algorithm is not well suited for retracking echoes departing from the Brown model, such as echoes in rain events, bloom events, marginal sea-ice $[4,22]$. In opposite, with the adaptive algorithm, the $\sigma^{0}$ spectrum is much more continuous. This is because, with the Adaptive model, the $\sigma^{0}$ parameter is constrained by the trailing edge and not only by the amplitude of the echo.

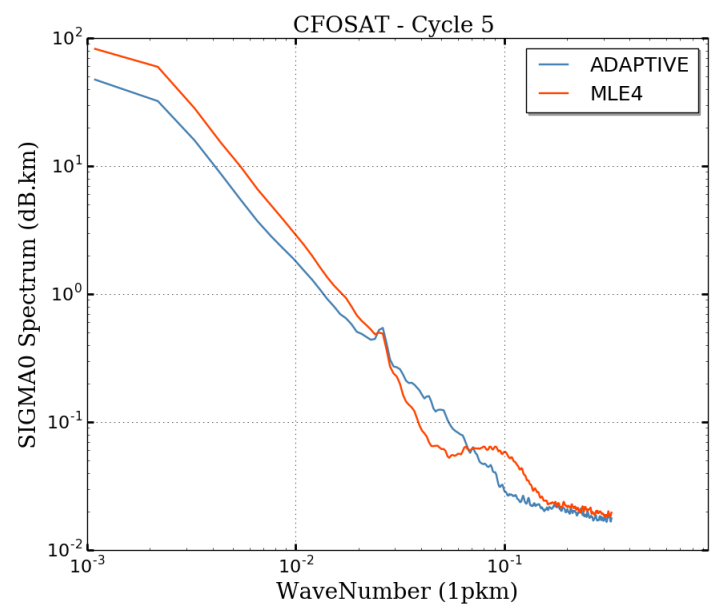

Figure 9: MLE4 and Adaptive $\sigma^{0}$ spectral analysis (MLE4 in orange, Adaptive in blue).
This improvement can be particularly well observed on special events such as a rain event as illustrated in Figure 10. On this example, we can see SWIM nadir echoes impacted by a rain event, characterized by a significant loss in the amplitude of waveforms associated with a decrease in the AGC (Automatic Gain Control) values compared to its classical oceanic value (19 $\mathrm{dB}$ for SWIM) [24]. Even though both $\sigma^{0}$ parameters are impacted by the rain event, the Adaptive estimates follow the AGC variations, whereas the MLE4 does not. In the Adaptive algorithm, the echo trailing edge is constrained by the mss (and hence $\sigma^{0}$ ), which enables $\sigma^{0}$ to follow naturally the amplitude variations of the signal, making it a much better tool compared to the MLE4 to detect specific events such rain as cells. Indeed, as explained in section III.B, the latter uses a model where the trailing edge of the echo is only constrained by the attitude of the altimeter. In specific events where the surface roughness dominates the trailing edge, the Brown model is thus not a suitable option. Note that a rain flag is defined in the nadir products, using [24]. The preliminary analysis already shows good consistency between this flag and collocated radiometers. As explained in [9], the introduction of a parameter related to the surface roughness allows the retracking of peaky echoes, characteristic of specular surfaces such as sea-ice. This is particularly of interest in the Arctic basin where the diversity of surface types can result in complex waveform shapes and characteristics. Indeed, leads (calm water) and polynyas (new sea ice) are characterized by a highly strong power return. On the other hand, a uniform cover of sea-ice will mainly have stronger signal than ocean, due to the high reflectivity of the sea-ice. But in some areas, the specificities of the ice will imply a received power similar to over ocean, typically at the west of the Arctic basin. The $\sigma^{0}$ values retrieved in the Arctic region are displayed in Fig. 11 for the MLE4 and Adaptive algorithms. Fig. 12 shows for the same period, the sea-ice concentration parameter extracted from ECMWF (Figure 12b) and the sea-ice type extracted from the OSISAF website for a specific day during the period (Figure 12a). As expected, the $\sigma^{0}$ estimated from MLE4 do not show a good consistency with the sea-ice characteristics: the retrieved $\sigma^{0}$ are lower over sea-ice than over the ocean and there is no variation visible in the Arctic basin. This is the consequence of the use of the Brown model, which was defined for fitting ocean-like waveforms only. 


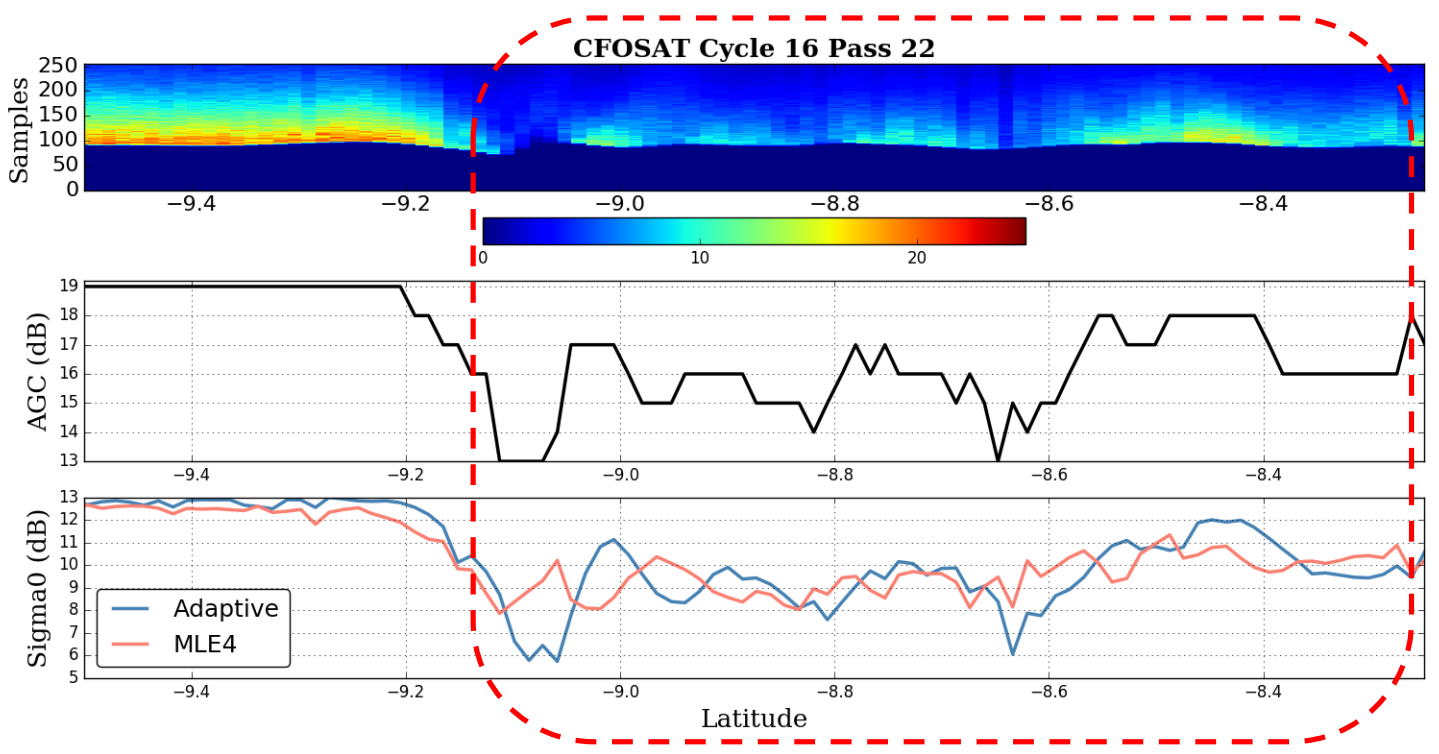

Figure 10 : From top to bottom: radargram illustrating power in each waveform bin for the chosen section along-track (color indicates intensity), Automatic Gain Control (AGC) for the same along-track section and $\sigma^{0}$ for the same along-track section (Adaptive in blue, MLE4 in red). The red dotted box indicates the beginning and end of the rain event.
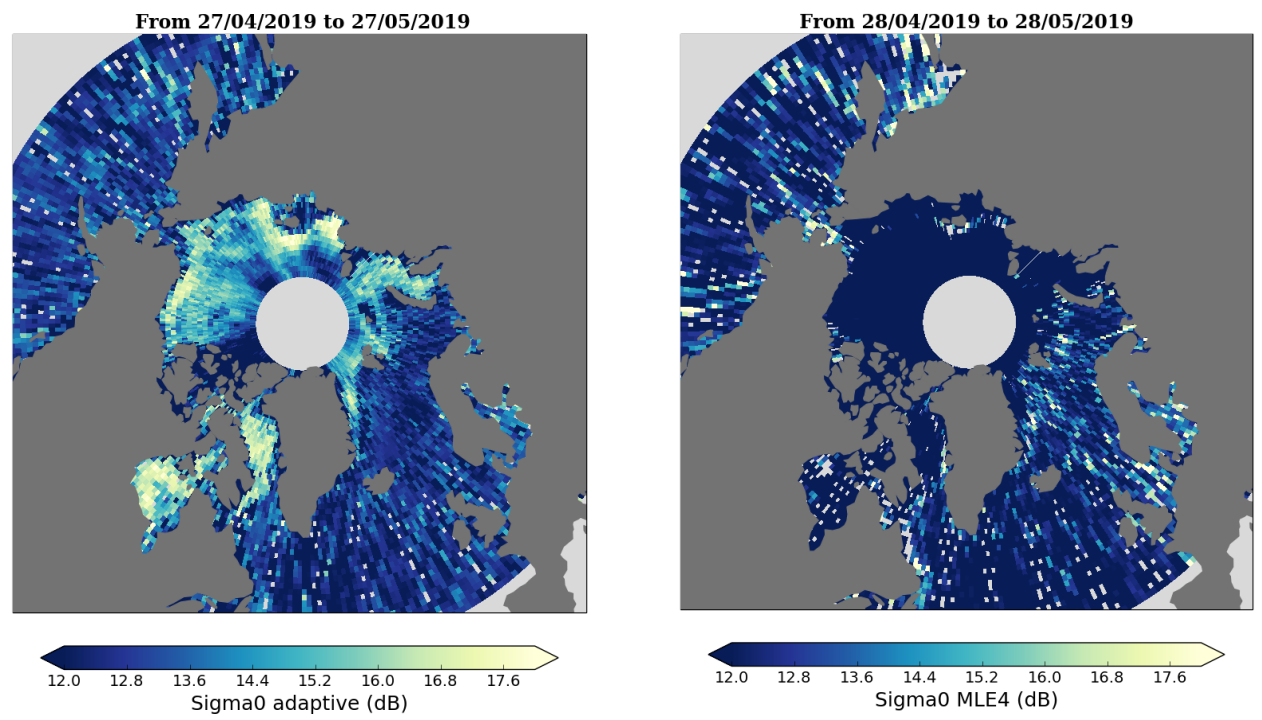

Figure 11: Gridded maps of $\sigma^{0}$ Adaptive (left) and $\sigma^{0}$ MLE4 (right) over 30 days of data 


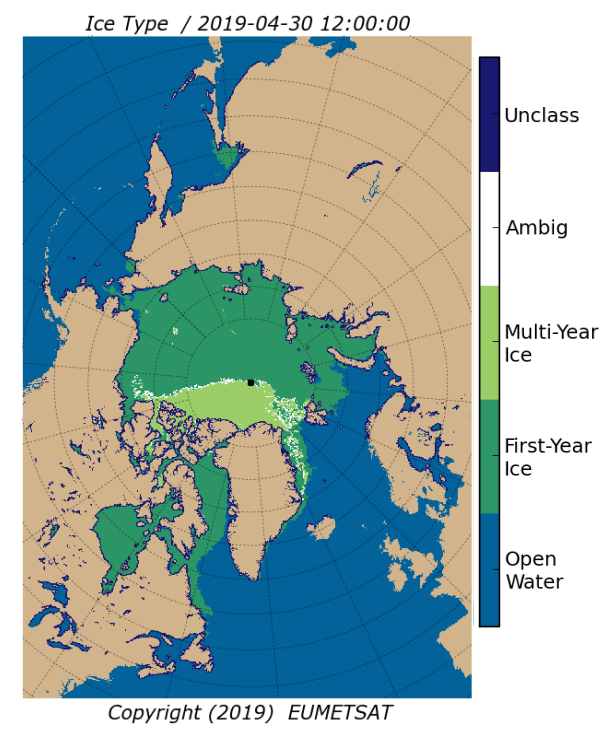

(a)

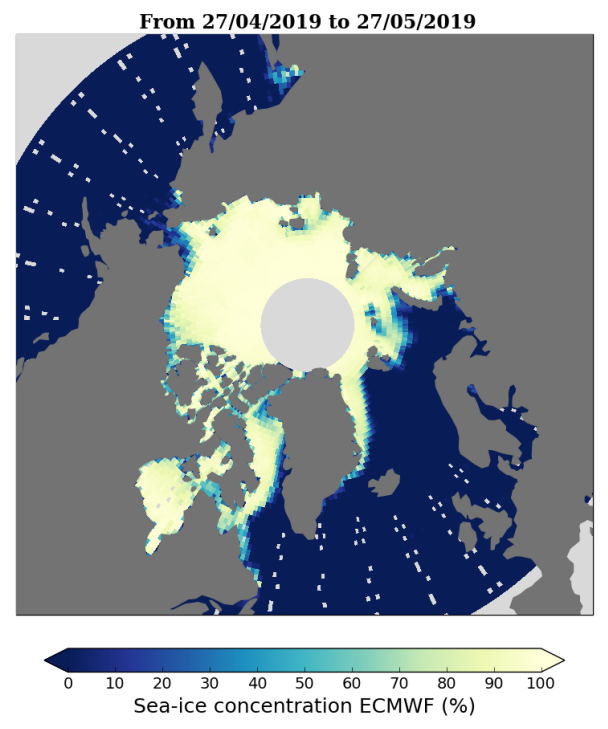

(b)

Figure 12 : Daily sea-ice type map extracted from the OSI-SAF quick-look website website http://osisaf.met.no/p/ for the day 30/04/2019 (a) and gridded map of sea-ice concentration from ECMWF over 30 days on data (b)

In opposite, the $\sigma^{0}$ obtained from the Adaptive retracker (Figure 11 left) shows a qualitative high consistency with the sea-ice extent (Figure 12b), and exhibits variations within the Arctic basin consistent with the ice type map of Figure 12a, with firstyear ice observed in Figure 12a corresponding to the highest values of $\sigma^{0}$. These preliminary results show that the Adaptive model fits well non-Brownian echoes. This opens perspectives for studies on sea-ice surfaces, as well as on scenes affected by rain and/or bloom. Indeed, thanks to its inclination, CFOSAT reaches a maximum latitude of 82.5 degrees north, making it a good candidate to study the polar areas. As CFOSAT does not give information about topography, it cannot be used to compute the sea-ice freeboard. However, as shown here above, $\sigma^{0}$ values have potential to characterize sea-ice type, which could lead to multiple sea-ice applications. For instance, it could be used to enrich existing altimeter sea-ice extent data to improve the quality of multi-mission sea-ice extent products. And this work could be linked to analysis on SWIM off-nadir data which also shows sensitivity to sea-ice type. One operational application would be to replace the current sea-ice detection based on ECMWF data by a SWIM nadir or combined nadir and off-nadir sigma 0 processing.

\section{QUALITY ASSESSMENT OF THE ADAPTIVE ALGORITHM USED FOR SWIM BASED ON INDEPENDENT DATA SETS}

To complete the assessment of the operational SWIM data products which are based on the adaptive algorithm, we analyze in this section the SWIM nadir data with respect to independent external datasets such as models, and conventional altimetric missions.

\section{A. Description of the dataset}

This assessment is based on SWIM L2 nadir products, in operational processing version V4.3.2, more specifically the following parameters given at 5Hz: swh_native and sigma0_native derived from the Adaptive algorithm, and wind native, derived from $\sigma^{0}$ thanks to the inversion algorithm described in [25]. Results include comparisons to models, interpolated at SWIM resolution as well as comparisons to altimetric missions (Jason-3 and AltiKa). For the latter, a colocation at crossover points was performed by retaining points when distances are less than $7 \mathrm{~km}$ along track and time differences are less than 3 hours. The cycles chosen for this analysis are the cycles 21 to 28 , covering the period from 2019 July $28^{\text {th }}$ to October $1^{\text {st }}$.

\section{B. Significant Wave Height (SWH)}

Figure 13 shows a $2^{\circ} \times 2^{\circ}$ gridded comparison of SWIM nadir SWH to ECMWF Hs model. As seen on the histogram on top of the plot, a good agreement is noticed, with a global bias of $\sim 3 \mathrm{~cm}$. The map highlights that the highest bias values can be observed in specific regions corresponding to low and high 
waves (high latitudes and Indian ocean mostly). Nevertheless, as already shown in Fig.6 of [13], the mean biases are less than $1 \mathrm{~cm}$ regardless of the sea-state and show a minor positive trend with wave height (from 0 to about $0.5 \mathrm{~m}$ for significant wave height varying from 2 to $7 \mathrm{~m}$ ) and wind speed (from 0 to $0.2 \mathrm{~m}$ for wind speed varying between 2 and $17 \mathrm{~m} / \mathrm{s}$ ). This wave height bias is negative for small significant wave heights (less than $1 \mathrm{~m}$ ), null around 2 meters height and positive for higher waves (Fig.6 of [13]).

\section{SWH Differences CFO / Model ECMWF}

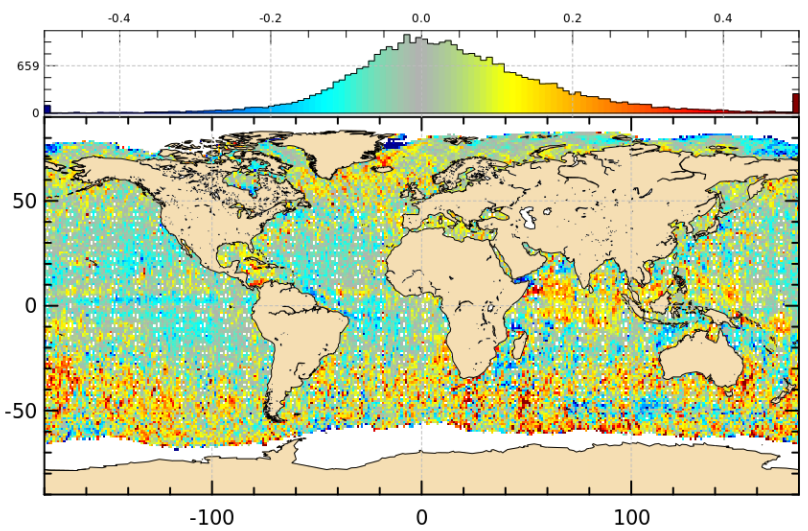

$\mathrm{m}$

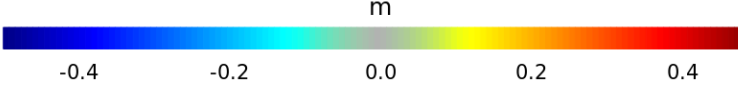

Figure 13 : Difference between SWIM nadir SWH and ECMWF Hs for SWIM cycles 21 to 24: Map and histogram (on top).

Mean $=0.0362 \mathrm{~m}$, Median $=0.0246 \mathrm{~m}$, Standard Deviation $=0.1478 \mathrm{~m}$

The comparison to altimetric data from Jason-3 and SARAL/AltiKa also shows a good agreement. Small biases with weak SWH dependencies can be observed in Figure 14, except for the points below $1 \mathrm{~m}$ and above $6 \mathrm{~m}$ where the number of crossover points are much smaller. This smaller number of points also induces an increase of the standard deviation around the mean. For this comparison, it is important to note that the SARAL and Jason-3 datasets are based on the MLE4 retracker, corrected using look-up tables, whereas SWIM estimates do not need any look-up tables as proved in sections IV and V.

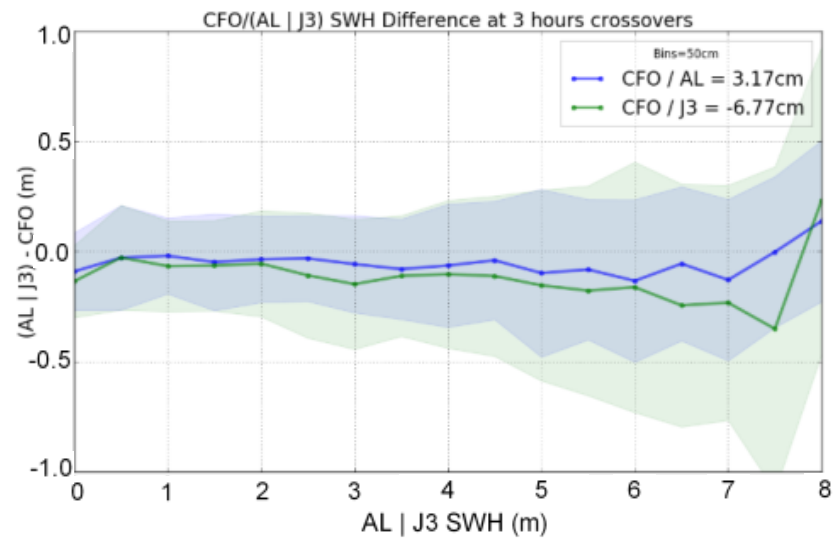

Figure 14: Bias between SWIM SWH and two altimeter missions (SARAL/AltiKa in blue, Jason-3 in green) for SWIM cycle 21 to 28. Each dot of the solid line is the mean computed per box of $50 \mathrm{~cm}$ of SWH and the envelope represents the associated standard deviation

\section{Backscattering coefficient $\sigma^{0}$ and wind speed}

As already described in [13], the analysis over data from cycle \#23 (from 2019 august $14^{\text {th }}$ to $26^{\text {th }}$ ) has shown the remarkable consistency of the $\sigma^{0}$ parameter between SWIM and Jason-3 altimeter (Poseidon-3B) which is in Ku-Band, as SWIM. A mean difference of only $0.12 \mathrm{~dB}$ (with $0.4 \mathrm{~dB}$ standard deviation) was found in this comparison.

On the other hand, when comparing SWIM results ( $\mathrm{Ku}$ band) to AltiKa results (in Ka Band), biases are observed, as described in [13]. They are mainly due to the different response of the backscattering in $\mathrm{Ku}$ with respect to Ka band.

As opposed to $\sigma^{0}$ (see just above) the winds retrieved from the $\sigma^{0}$ (through empirical relationships relating $\sigma^{0}$ to wind speed and SWH) are directly comparable between altimeters, even for different radar bands, provided that the empirical models used for converting $\sigma^{0}$ into wind speed have been assessed independently. Here we compare SWIM wind to ECMWF model outputs, AltiKa and Jason-3 data. The wind speed obtained with SWIM is consistent with the ECMWF model wind speed (Figure 15). The mean difference is $0.32 \mathrm{~m} / \mathrm{s}$, and the standard deviation of the difference is $0.92 \mathrm{~m} / \mathrm{s}$. The highest differences are observed in the tropics and at high latitudes. For these areas of extreme Sigma0 values (either very small or high), the differences are likely due to three mixed effects. First, a too smooth ECMWF model that does not see all the short scale variability (mostly in the intertropical zone). Then, a higher uncertainty on wind inversion for poorly represented numerical populations. A new inversion model is planned to be computed again on a longer period to lower this later impact. Finally, although a data selection was applied, some mixed area (sea ice and/or rain) may still locally impact data. This will be further studied and should be reduced in future product version.

Wind Differences CFO / Model ECMWF

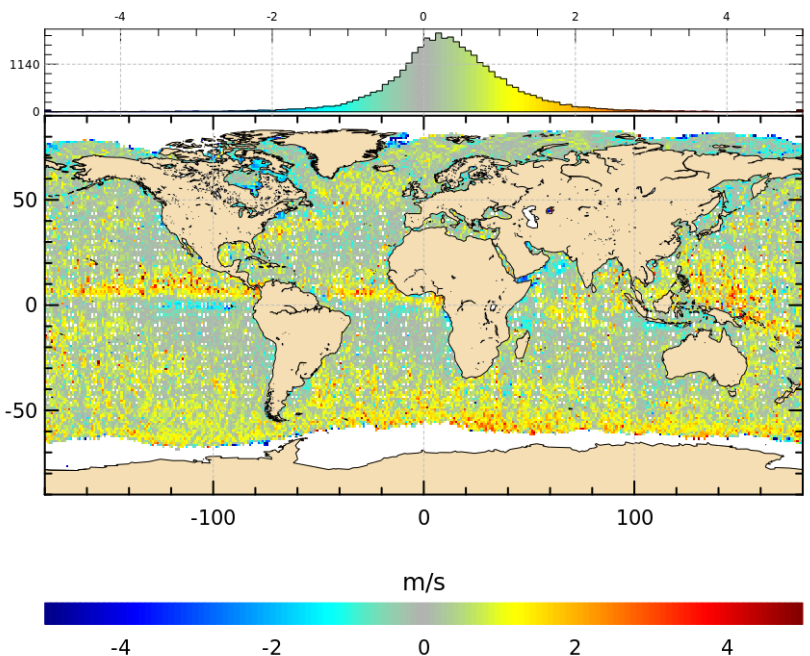

Figure 15: Difference between SWIM nadir altimeter wind and ECMWF wind for SWIM cycle 21 to 28 Map and histogram (on top). Mean $=0.32 \mathrm{~m} / \mathrm{s}$, Median $=0.30 \mathrm{~m} / \mathrm{s}$ and Standard Deviation $=0.92 \mathrm{~m} / \mathrm{s}$

Compared to the winds provided by Jason-3 and SARAL/AltiKa at crossover points (within 3 hours and $7 \mathrm{~km}$, as for Figure 14), the winds from SWIM are quite consistent as 
shown in (Figure 16). The mean differences (around $-1 \mathrm{~m} / \mathrm{sec}$ ) can be attributed to the $\sigma^{0}$ inversion retrieval algorithms because they differ for each mission. Furthermore, for extreme data (below $1 \mathrm{~m}$ and above $6 \mathrm{~m}$ ), the amount of data is much weaker and the metrics though, less stable. For AltiKa, the algorithm is based on [26], whereas for SWIM it is estimated from [25]. In the future, a refinement of the model [25] will be proposed based on a $2 \mathrm{D}$ look-up table $\left(\sigma^{0}\right.$ function of wind speed and significant wave height) as proposed in [27]. It will be carried out using one year of SWIM data to ensure a stable statistical model.

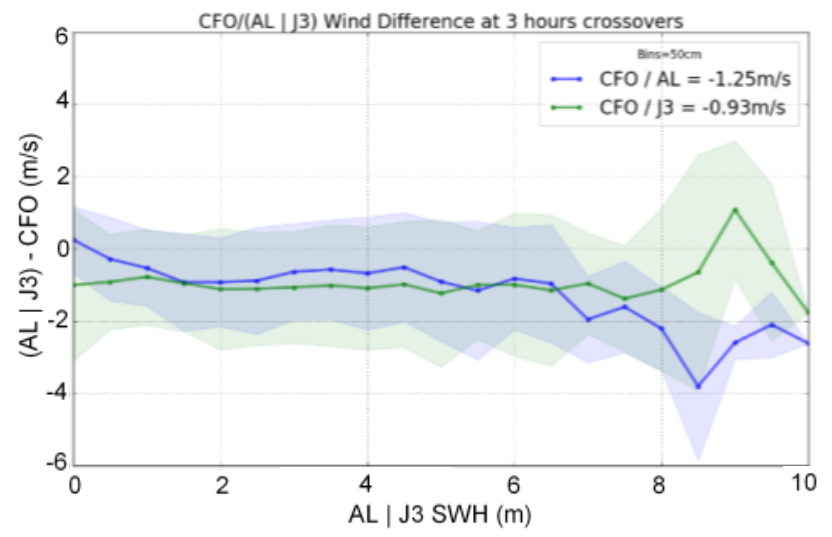

Figure 16 : Bias between SWIM wind and two altimeter missions (SARAL/AltiKa in blue, Jason-3 in green) for SWIM cycle 21 to 28. Each dot of the solid line is the mean computed per box of $50 \mathrm{~cm}$ of SWH and the envelope represents the associated standard deviation.

\section{SUMMARY AND CONCLUSION}

In this paper we have described and validated the Adaptive algorithm proposed to retrieve the significant wave height and the normalized radar cross-section from radar nadir echoes. We have shown from simulations that compared to the classical MLE4 algorithm, these parameters are retrieved without bias and with less noise (restitution noise reduction of $60 \%$ for SWH and $11 \%$ for $\sigma^{0}$ ). As it is not an output of the CFOSAT product, range performances from the Adaptive algorithm have not been detailed in this paper. However, significant improvements are also observed for this parameter on oceanic echoes [28], among other, we can note a $10 \%$ restitution noise reduction, without any bias.

When applied on real CFOSAT/SWIM data, these improvements ensure the same level of performance over ocean than conventional altimetry missions, in spite of the SWIM instrument lower measurement rate $(4.5 \mathrm{~Hz}$ vs $20 \mathrm{~Hz})$. This indicates that for a given noise level on the raw echoes, the geophysical variability can be detected with the Adaptive algorithm at smaller scales than with the MLE4 standard method. This opens the way to higher resolution data analysis from conventional altimetry missions, which will fulfill the need of end-users to refine regional models at small scales.

Despite its increased complexity (in particular the numerical convolution with the real Point Target Response), the processing with the Adaptive retracking method is compliant with near real-time production requirements. For example, for CFOSAT, the processing time is approximately 3 minutes for 105 minutes of measurements. Other missions could follow this upgrade to improve the whole nadir constellation. As a matter of fact, the Jason future reprocessed products will include products derived from the Adaptive algorithm as an alternative to the MLE4. Both solutions will be available in the products.

In addition, we have shown that the Adaptive Algorithm provides improved parameters on specific areas such as sea-ice or rain impacted surfaces, mainly due to the choice of the Amarouche flat surface response model. This latter introduces the mean square slope of the surface as a parameter of the flat response, allowing to better invert echoes that are different from the Brown model shape. Although no analysis has been done yet on the retrieved mean square slope parameter, one can presume that it will bring complementary information on the surface roughness characterization, especially for areas where the surface roughness changes rapidly, typically in sea-ice regions, inland waters such as rivers, and specific events such as rain cells and blooms. Thanks to the improvement of the retrieved parameters, new applications could also be considered over sea-ice surfaces. CFOSAT SWIM nadir products offer a large dataset to explore the potential of this processing.

Finally, it should be mentioned that although this study was specifically conducted under the SWIM instrument configuration (simulations) and on SWIM real data, several conclusions are more general and should be considered for improvements in future altimeter data processing or reprocessing. In particular: the use of real PTR, allowing the suppression of look up table; and the use of a likelihood estimator instead of a least square estimator, leading to a reduction of the restitution noise.

\section{ACKNOWLEDGMENT}

The authors would like to thank all contributors not mentioned in the list of co-authors: M. Dalila for all her work on the SWIM databases used for the data analysis presented in this paper, F. Andzembe for his implication in the development of the processing chain, N. Tran for her contribution with the wind analysis, L. Amarouche who developed the model used by the Adaptive algorithm, and F. Gouillon for its work and efficiency as the ground segment development manager.

\section{REFERENCES}

[1] G. S. Brown, "The average impulse response of a rough surface and its applications", IEEE Trans. Antennas Propagai., vol. AP25, pp. 67-74, Jan. 1977.

[2] J.-P. Dumont, "Estimation optimale des paramètres altimétriques des signaux radar Poséidon," Ph.D. dissertation, Univ. Toulouse-ENSEEIHT, Toulouse, France, 1985.

[3] L. Amarouche, P. Thibaut, O. Z. Zanife, J.-P. Dumont, P. Vincent, and N. Steunou. "Improving the Jason-1 ground retracking to better account for attitude effects". Marine Geodesy, 27(1-2): pp 171-197, Aug. 2004.

[4] P. Thibaut, J. C. Poisson, E. Bronner, and N. Picot, "Relative performance of the MLE3 and MLE4 retracking algorithms on jason-2 altimeter waveforms". Marine Geodesy, 33(sup1): pp 317-335, March 2010.

[5] D.J Wingham, C.G Rapley, H. Griffiths (1986). "New techniques in satellite altimeter tracking systems". Proceedings of IGARSS'86 Symposium, Zürich, 8-11 Sept. 1986, Ref. ESA SP-254, 1339-1344

[6] F. Frappart, S. Calmant, M. Cauhopé, F. Seyler, A. Cazenave, «Preliminary results of ENVISAT RA-2-derived water levels validation over the Amazon basin". Remote Sensing of Environment, Elsevier, 2006, 100 (2), pp.252-264. 10.1016/j.rse.2005.10.02. 
[7] B. Legresy, F. Papa, F. Remy, G. Vinay, M. Van den Bosch., O. Zanife (2005). "ENVISAT radar altimeter measurements over continental surfaces and ice caps using the ICE-2 retracking algorithm". Remote Sensing of Environment, 95 (2), 150-163. ISSN 0034-4257

[8] M. Passaro,, P. Cipollini, S. Vignudelli, G.D. Quartly, H.M. Snaith, "ALES: A multi-mission adaptive subwaveform retracker for coastal and open ocean altimetry", Remote Sensing of Environment 145:173-189 . April 2014.

[9] J-C Poisson, G Quartly, A. Kurekin, P. Thibaut, D.Hoang and F.Nencioli , "Development of an ENVISAT Altimetry Processor Providing Sea Level Continuity Between Open Ocean and Arctic Leads", IEEE Transactions on Geoscience and Remote Sensing, Vol. 56, Issue 9, pp 5299 - 5319 ,Sept. 2018.

[10] D. Hauser, D. Xiaolong ; L. Aouf ; C. Tison ; P. Castillan, "Overview of the CFOSAT mission", IGARSS'2016, Beijing (China), July 2016

[11] D. Hauser, C. Tison, T. Amiot, L. Delaye, N. Corcoral et al, SWIM: the first spaceborne wave scatterometer, IEEE Trans. on Geoscience and remote Sensing, 10.1109/TGRS.2017.2658672, VOL 55, 5, May 2017

[12] Dong Xiaolong, Zhu Di, W. Lin, H. Liu, J. Jiang, "A Ku-band rotating fan-beam scatterometer: design and performance simulations", IGARSS'10, 2010

[13] D. Hauser, C. Tourain, L. Hermozo, D. Alraddawi, L. Aouf, B. Chapron, A. Dalphinet, L. Delaye, V. Gressani, A. Grouazel, G. Guitton, R. Husson, A. Mironov, A. Mouche, A. Ollivier, L. Oruba, F. Piras, R. Rodriguez Suquet, P. Schippers, C. Tison, Radar observation of surface ocean waves and wind from space: first results from the SWIM instrument on-board CFOSAT, IEEE Transaction on Geoscience and Remote Sensing, 10.1109/TGRS.2020.2994372, 2020

[14] P. Thibaut, L. Amarouche, O. Z. Zanife, N. Steunou, P. Vincent, and P. Raizonville. "Jason-1 altimeter ground processing look-up correction tables", Marine Geodesy, 27(3-4): pp 409-431, July 2004.

[15] G. Hayne, "Radar altimeter mean return waveforms from near-normalincidence ocean surface scattering", IEEE Trans. Antennas and Propagat. vol.: 28, Issue: 5, pp. 687 - 692 Sep 1980

[16] Amarouche L. (2001) : "Contributions à l'Etude du Biais d'Etat de Mer", PhD Thesis, UNIVERSITE PARIS 7 -DENIS DIDEROT L. Amarouche, E. Thouvenot, B. Chapron, and O.-Z. Zanife, "A newestimator of the sea state bias using a three frequency radar altimeter". In Proc. IEEE Int. Conf. Geosci. and Remote Sensing (IGARSS), pp 2510-2512, Sydney, Australia, July 9-13, 2001.

[17] J. MacArthur and J. H. U. A. P. Laboratory." SEASAT - a radar altimeter design description". Johns Hopkins University Applied Physics Laboratory, 1978.

[18] L. Amarouche, S. Jourdain, and J. R. Deboer, "Rapport Tâche 2.2: Retracking Spécifique Cellules de pluie et Blooms," CNES, Toulouse France, Tech. Rep. SALP-NT-P-EA-21776-CLS, 2010.

[19] L. Amarouche and A. Vernier, "New methods for retracking altimeter sea ice and ice sheets waveforms," ESA, Toulouse France, Tech. Rep. CLSDOS-NT-10-192, 2011.

[20] A. Halimi : "From conventional to delay/Doppler altimetry", $\mathrm{PhD}$ thesis, Institut National Polytechnique de Toulouse, Toulouse, France, 2013.

[21] J.Nelder and R. Mead, " A simplex method for function minimization », Computer Journal, vol. 7, no 4, 1965, p. 308-313

[22] P.G, Chalenor and M. A Srokoez. 1989. "The extraction of geophysical parameters from radar altimeter returns from a non-linear sea surface. In Mathematics in remote sensing", ed. S. R. Brooks. Oxford: Clarendon Press.

[23] G. Dibarboure, F. Boy, J.D. Desjonquères, S. Labroue, Y. Lasne, N. Picot, J.C. Poisson and P. Thibaut, "Investigating short-wavelenght correlated errors on low-resoltion mode altimetry", Journal of Atmospheric and Oceanic Technology, June 2014.

[24] J.Tournadre, J.C. Poisson, N.Steunou and B.Picard, "Validation of AltiKa Matching Pursuit Rain Flag", Marine Geodesy, 38-1, p 107-123, 2015, doi10.080/01490419.2014.1001048

[25] Gourrion, J., D. Vandemark, S. Bailey, B. Chapron, G. P. Gommenginger, P. G. Challenor, and M. A. Srokosz: A two-parameter wind speed algorithm for Ku-band altimeters. J. Atmos. Oceanic Technol., 19, 20302048, 2002, doi:10.1175/1520-0426.

[26] J. Lillibridge, R. Scharroo, S. Abdalla, and D. Vandemark, 2013, One-and Two-DimensionalWind Speed Models for Ka-band Altimetry,Journal of Atmospheric and Oceanic Technology,doi :http://dx.doi.org/10.1175/JTECH-D-13-00167.1
[27] F.Collard. "Algorithmes de vent et période moyenne des vagues JASON à base de réseaux de neurones". Boost Technologies. BO-021-CLS-0407RF, 2005.

[28] "Adaptive retracker for conventional altimeter echoes: physical basics and main performances obtained on Jason-3 GDR-F dataset", to be submitted" 